\title{
LES BESOINS ET L'ORGANISATION DE FORMATIONS COMPLÉMENTAIRES POUR LES DOCTORANTS EN EEA
}

\author{
Olivier Bonnaud \\ Collège des Ecoles Doctorales de Rennes, Université de Rennes 1, \\ 35042 Rennes Cedex France \\ olivier.bonnaud@univ-rennes1.fr
}

\begin{abstract}
Résumé : Depuis la mise en place du processus de Bologne en Europe cherchant à harmoniser les études supérieures et depuis l'arrêté Ministériel du 07 août 2006 relatif aux études doctorales, de façon analogue à la plupart des pays développés (anglo-saxons et de l'extrême orient) les études doctorales correspondent d'une part à la préparation de la thèse et d'autre part à des enseignements complémentaires. Ces formations constituent un complément au diplôme mais aussi doivent préparer le futur docteur à la vie active aussi bien dans les organismes de recherche et d'enseignement supérieur que dans les centres de recherche et développement des entreprises. Cette présentation rappelle les besoins en compétences et savoir-faire des docteurs, précise les formations et la gestion des prises en compte au niveau des écoles doctorales, et donne des exemples de formations réellement organisées surtout dans le domaine de l'EEA.
\end{abstract}

Mots clefs: Etudes doctorales, harmonisation européenne, formations professionnalisantes, scientifiques

\section{INTRODUCTION}

La mise en place du processus de Bologne en Europe [1] a eu pour but d'harmoniser les études supérieures en Europe suivant un schéma Bachelor, Master et Doctorat [2] ou 3-5-8 afin de faciliter les échanges des diplômés intra-européens. Le processus a été engagé en France dès l'année 2000 pour le niveau licence ou Bachelor et en 2003 pour le niveau master. En 2006, l'arrêté Ministériel du 07 août relatif aux études doctorales [3] redéfinit la formation de docteurs avec une approche analogue à la plupart des pays développés (anglo-saxons et de l'extrême orient). Dans cette nouvelle définition, les études doctorales correspondent d'une part à la préparation de la thèse, et d'autre part à des formations ou enseignements complémentaires dispensés en cours d'études doctorales. Ces formations constituent d'une part la matière à un complément au diplôme et d'autre part doivent préparer le futur docteur à la vie active aussi bien dans les organismes de recherche et d'enseignement supérieur que dans les centres de recherche et développement des entreprises. Après un rappel sur les besoins en compétences et savoir-faire des docteurs, le document présente l'organisation et la nature des formations. Une attention particulière est portée au domaine de l'EEA.

\section{COMPÉTENCES EXIGÉES DES DOCTEURS}

$\mathrm{La}$ formation de docteurs correspond à l'acquisition de compétences scientifiques généralement dans le domaine spécifique de la thèse, sachant que des situations d'interdisciplinarité sont de plus en plus fréquentes, mais aussi à une efficacité dans la production scientifique, de l'innovation et la maîtrise du transfert vers les entreprises, et enfin des qualités personnelles (cf. figure 1). Citons parmi ces dernières :

- la capacité d'analyse et de synthèse,

- l'aptitude à approfondir une connaissance aussi bien à l'aide d'une compilation bibliographique que par des expérimentations originales,

- le management de la recherche,

- la direction scientifique d'une petite équipe,

- une autonomie dans la démarche et la recherche de financement pour assurer une activité de recherche [4].

Cela sous-entend une adaptation du candidat à une équipe de recherche dans laquelle il assume un comportement et une démarche scientifiques, mais aussi des compléments de formations de compétences dans le domaine des sciences « douces », telles la maîtrise de langues étrangères, la conduite de projet, la connaissance des entreprises.

Ces besoins sont aussi en liaison avec la nature des emplois des docteurs qui dans le domaine de l'EEA se placent pour un tiers d'entre eux dans les structures de recherche académiques ou des organismes nationaux de recherche mais pour deuxtiers dans les services ou départements de recherche et développement des entreprises [5].

\section{MISE EN PLACE DES FORMATIONS}

\section{III.1 Nature des formations}

Durant la préparation de leur thèse, les doctorants doivent suivre des formations qui se répartissent dans les 3 domaines suivants : 


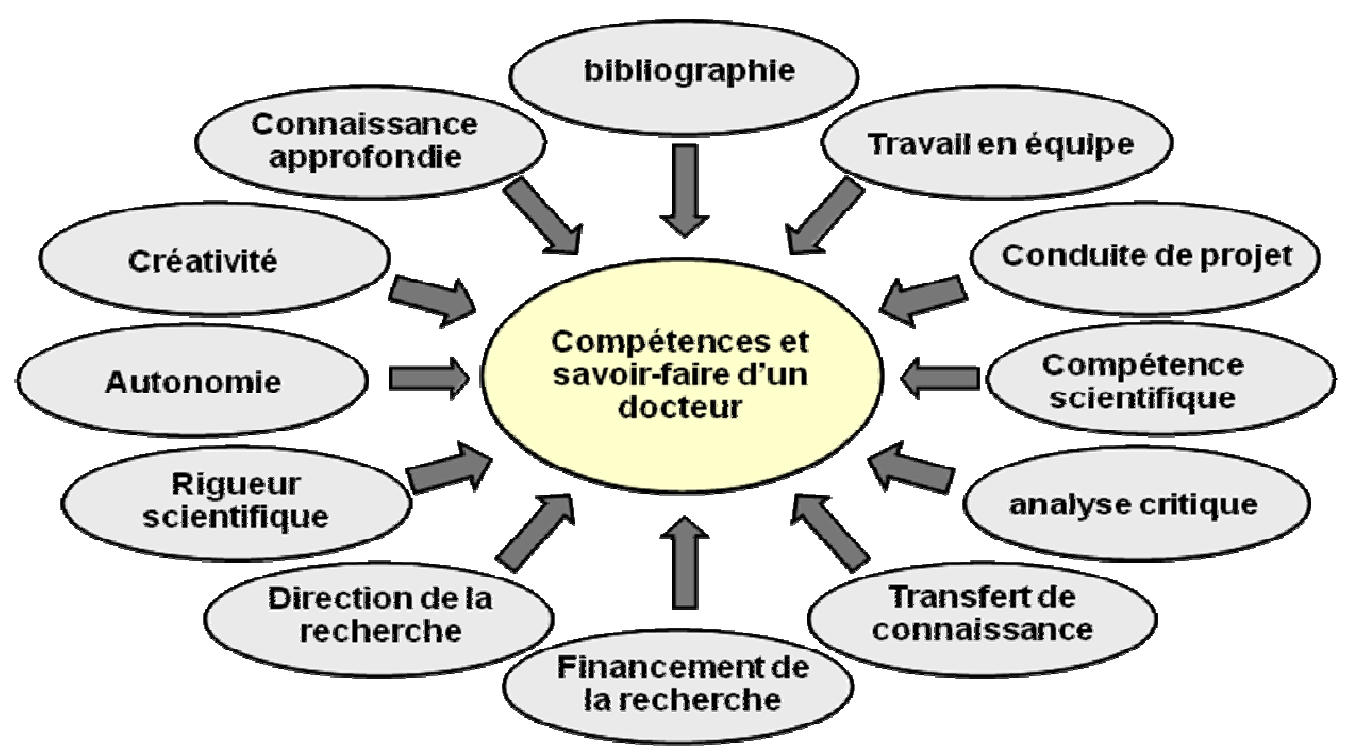

Figure 1: Représentation schématique des compétences qui peuvent être exigées des docteurs. Ces compétences sont valables pour l'ensemble des disciplines. Elles sont de plus en plus exigées par les recruteurs.

- cours scientifiques, cours de haut niveau, keynotes, écoles d'été ou écoles d'hiver (summer or winter schools), journées nationales ou régionales de doctorants, cours des domaines généraux et de sciences douces (formation générale et linguistique), cours à visée professionnelle ou professionnalisants.

Ces trois catégories sont réparties avec un certain équilibre (1/3 chacune par exemple).

Les écoles doctorales ont constitué un référentiel de formation correspondant à des crédits. Ces crédits sont en relation avec des heures de formation suivies dans la plupart des écoles. Le nombre de crédits dépend des écoles et des disciplines. Par exemple, le nombre total minimal de crédits requis est de 50 pour l'école doctorale MATISSE [5-6] correspondant aussi à 50 heures équivalentes de formation. Il faut noter que ces crédits ne sont pas des ECTS (European Credit Transfer System) puisque dans ce cas, ces crédits ne sont pas des équivalences de modules acquis pouvant être validés par un autre établissement dans le cadre d'échanges (ERASMUS par exemple). Dans le cadre de thèses en cotutelle, ils peuvent toutefois être pris en compte par les partenaires étrangers, si la convention de cotutelle a été établie avec précaution.

L'équivalence d'heures est basée sur une quantité de travail qui pourrait être fournie par un doctorant dans le cas d'un cours de nature scientifique avec du travail supplémentaire personnel. L'exemple le plus fréquent est la contribution à l'organisation d'une manifestation (telle que conférence, journée régionale ou nationale de doctorants, etc.) pour laquelle par exemple une dizaine de crédits (ou heures) peuvent être attribués pour une implication réelle dans le comité d'organisation. Cela constitue aussi un moyen d'encourager les doctorants à s'impliquer dans ces tâches qui sont formatrices mais pas toujours valorisées.

\section{2 Complément au diplôme}

Ces formations et leur intitulé apparaissent dans le complément au diplôme qui est préparé et maintenant, pour la plupart des écoles doctorales, exigé pour la demande d'autorisation de soutenance. Il faut savoir que ceci correspond aux vœux du Ministère, mais aussi à une pratique déjà courante dans certains pays ayant adopté le modèle anglosaxon. Ce complément au diplôme a donc une importance immédiate, mais surtout pour la future carrière du docteur qui pourra faire état de son ouverture d'esprit, de ses connaissances scientifiques et de l'acquisition de compétences complémentaires dans les sciences douces, ce point étant de plus en plus apprécié au moment de l'embauche par les services des ressources humaines des entreprises.

Il est clair que pour les doctorants en entreprise et bénéficiant d'une bourse du Ministère de l'Industrie de type CIFRE, en cotutelle (entre deux établissements étrangers), ou salariés, des conditions particulières prenant en compte leur propre environnement ou leur plan de carrière sont applicables. Ils peuvent de plus bénéficier de formations propres aux entreprises (par exemple stage de formation à l'hygiène et à la sécurité) qui peuvent aussi être pris en compte dans le récapitulatif établi par l'école doctorale.

Il peut être noté qu'encore de trop nombreux directeurs de thèse, même s'ils deviennent très minoritaires, considèrent ces formations comme du temps perdu vis-à-vis de l'activité purement 
scientifique centrée sur le sujet de thèse. Au niveau de la direction des écoles doctorales, des efforts sont encore fournis pour convaincre de l'intérêt, principalement pour le futur docteur. En pratique, des courriers de rappel fréquents sont envoyés aux doctorants afin qu'ils remplissent les conditions minimales de formations.

\section{IV.FORMATIONS ASSURÉES}

\section{VI.1 Formations liées à la recherche}

Ce sont des cours d'initiation à un domaine de recherche plus spécialisés que les cours de Master, ou des cours à caractère interdisciplinaire. Ils se placent au niveau des doctorants et s'adressent à un public plus large que les séminaires des équipes de recherche. Ces cours complètent ainsi pour les doctorants la formation acquise en Master (ou école d'ingénieurs) et peuvent apporter une ouverture sur d'autres domaines de recherche.

Chaque année, des cours spécialisés de haut niveau à audience internationale sont organisés et soutenus financièrement par les écoles doctorales, et les collèges doctoraux régionaux (quand ils existent). Quelques exemples de titre de ces cours sont donnés ci-dessous :

- la radio logicielle,

- nouvelle approche de la fiabilité,

- les antennes,

- outils informatiques,

- composants actifs en électronique de puissance

- développement durable et génie électrique et électronique,

- fiabilité et maintenance industrielle,

- commande et régulation adaptative,

- perception visuelle

- compatibilité électromagnétique

- diffraction des rayons X par les poudres

- perspectives en microélectronique, concept de nouveaux dispositifs

- composants innovants pour l'intégration ultime sur silicium.

Dans la même catégorie sont prises en compte les écoles d'été, les écoles d'hiver qui sont vivement conseillées. L'école doctorale et les équipes d'accueil des doctorants peuvent contribuer au financement pour la participation à ces cours.

VI.2 Cours de Master ou de $3^{\text {ème }}$ année d'école d'ingénieurs

Les cours donnés dans les Masters ou dans les $3^{\text {ème }}$ année d'écoles d'ingénieurs des établissements en accréditation conjointe ou associés à l'établissement porteur des écoles doctorales, offrent également aux doctorants des possibilités de formation sur des thèmes complémentaires à leur discipline principale, en réponse aux besoins d'acquisition de compétences. Par exemple des compléments de mathématiques aux doctorants en économétrie, ou des cours de technologie microélectronique à des électroniciens ou des électrotechniciens, voire des biologistes ou des chimistes en vue de développer des capteurs. Des exemples de ces cours peuvent être trouvés sur les sites web des écoles doctorales. Par exemple, la liste exhaustive de cours communs aux masters et $3^{\text {ème }}$ année d'école d'ingénieurs est donnée sur le site de l'école doctorale EEATS de l'INP Grenoble [7]. Dans ce cas, un livret des formations est constitué et permet aux doctorants de choisir cellesci en fonction de leur projet professionnel ; parmi les exemples de la liste ci-dessus, certains ont été sélectionnés dans cette école doctorale.

\section{VI.3 Cours professionnalisants}

Ces formations sont destinées à familiariser le doctorant avec le monde socio-économique. Les thématiques les plus fréquemment proposées sont les suivantes :

- connaissance de l'entreprise,

- création d'entreprises,

- les différentes formes structurales d'exercice d'une activité professionnelle,

- marché des capitaux et financement des entreprises,

- stratégie d'entreprise,

- stratégie internationale et compétitivité,

- innovation, création, invention,

- entreprise et environnement,

- entreprise et société,

- propriété industrielle,

- intelligence économique,

- analyse de la valeur,

- conduite de projet,

- techniques d'expression orale et recherche d'emploi,

- management et communication interne,

- veille technologique,

- gestion des Ressources Humaines,

- $\quad$ etc...

Cette liste déjà étendue mais pourtant non exhaustive est issue de l'analyse de plusieurs écoles doctorales du domaine de l'EEA (collège doctoral de Rennes, EEATS Grenoble, SPI Lille, SPI Bordeaux) et montre que ce type de formation est d'une part au menu de la plupart des écoles doctorales et d'autre part qu'il répond à des besoins exprimés par les milieux susceptibles de recruter les docteurs.

Il peut être noté qu'une partie du programme des doctoriales ${ }^{\circledR}$ organisées par les collèges doctoraux [8] depuis le milieu des années quatrevingt dix inclut cette approche. Cette manifestation correspond à un séminaire résidentiel de plusieurs jours portant notamment sur l'ensemble des formations professionnalisantes. 


\section{VI.4 Cours de formation générale}

Ces formations sont essentiellement destinées à élargir la culture générale des doctorants. La première demande est linguistique. Sachant qu'environ la moitié des doctorants étudiant en EEA est d'origine étrangère (par exemple $c f$.[4-5]), une majorité d'entre eux exprime le besoin d'une amélioration de la pratique de la langue française. Pour cela, les écoles doctorales doivent avoir une politique volontariste avec les établissements de rattachement par la prise en charge de ces formations. Cet aspect s'inscrit naturellement dans les politiques internationales des établissements et ne semble pas poser de problèmes dans les cas analysés.

Pour une autre part importante des doctorants du domaine de l'EEA, une reconnaissance de faiblesses dans la maîtrise de la langue anglaise qui est obligatoirement utilisée pour les publications scientifiques appelle à l'organisation de cycles de perfectionnement. Des cours de communications en anglais sont aussi régulièrement demandés, proposés et suivis, afin d'améliorer la communication orale et écrite scientifique. Ces formations sont en général très demandées par plus de la moitié des doctorants et les écoles doctorales doivent organiser plusieurs sessions chaque année.

Dans le domaine de l'EEA très peu de demandes pour d'autres langues étrangères sont en fait manifestées. Toutefois, des langues asiatiques commencent à être demandées, chinois et japonais principalement.

Des formations de sciences humaines, telles que la psychologie, les sciences de l'éducation, ou des formations pratiques comme l'hygiène et la sécurité ou le secourisme sont aussi dispensées et complètent le domaine des formations générales.

\section{CONCLUSION}

Le monde du travail accessible aux docteurs qui n'est plus limité au monde académique comme dans les années 60 et 70 a exprimé des besoins d'ouverture pour les scientifiques du domaine des hautes technologies vers les disciplines des sciences douces. L'évolution des études doctorales répond à ces besoins. Dans le domaine de l'EEA, des formations complémentaires sont ainsi proposées aussi bien dans les spécialités traditionnelles du domaine que dans d'autres approches d'ouverture intellectuelle et de connaissance des milieux professionnels. L'objectif global est l'acquisition de savoir-faire et de compétences attachées à la maîtrise d'une approche scientifique, d'innovation de transfert et de management. Ces qualités des docteurs sont de plus en plus requises au moment du recrutement. Des représentants des ressources humaines de grandes entreprises qui recherchent des docteurs ont fait part de leur grande sensibilité à ces différents aspects qui sont pris en compte dans les critères de recrutement.

Ces approches sont maintenant inclues dans l'évaluation des écoles doctorales par l'Agence Nationale (AERES, [8]), et constituent aussi un thème d'étude au sein de l'association européenne EAEEIE [9] et du réseau européen ELLEIEC [10] venant à la suite du réseau EIE-Surveyor [11]. Resterait à harmoniser les écoles doctorales au niveau national, voire européen, afin d'avoir une équivalence de crédits et des compléments au diplôme très semblables. Ce complément de formations peut aussi intégrer la formation tout au long de la vie. C'est l'une des actions qui sont actuellement menées au sein du réseau européen ELLEIEC [10] auquel participent des membres de la commission internationale du club EEA [12].

\section{RÉFÉRENCES}

[1] The Bologna Declaration on the European space for higher education: an explanation, 1999. [online]: http://europa.eu.int/comm/ education/policies/educ/bologna/bologna.pdf

[2] J.M. Thiriet et al., Overview of Bologna Process Implementation in Europe In Electrical and Information Engineering, Surveyor report, New Trends of Doctoral Studies in Europe: Special Considerations for the Field of Electrical and Information Engineering, ISBN 29516740-3-1, pp.15-21, 2009

[3] Decree of $07^{\text {th }}$ August 2006 by Education Ministry, published at Official Journal of French Republic on $24^{\text {th }}$ August, 2006

[4] O. Bonnaud, M. Hoffmann, New challenges of doctoral studies in Europe in the field of electrical and information engineering, Bologna Handbook. C4-4-4, $\mathrm{N}^{\circ} 12,2009 . \mathrm{Pp} 2-23$

\section{[5] http://www.matisse.univ-rennes1.fr}

[6] O. Bonnaud, Accreditation and functioning of French doctoral schools following the Bologna process in Electrical and Information Engineering, Oral communication; EAEEIE'06; Craiova (Romania) ; 1-2 June 2006, pp. 361-366.

[7] http://edeeats.grenoble-inp.fr/(formation-pendant-lathèse)/ ou consulter le site collège doctoral de Rennes : http://college-ed.univ-rennes.eu/theme/ Doctoriales/

[8] The new French Agency for Assessment of Research (AERES) is now operational. http://www.ambafranceau.org/article.php3?id_article $=2227$

[9] European Association for Education in Electrical and Information Engineering, http://www.eaeeie.org

[10] ELLEIEC: Enhancing Lifelong Learning for the Electrical and Information Engineering Community. Project Nr: 142814-LLP-1-2008-FR-ERASMUS-ENW.

[11] EIE-Surveyor, reference point for electrical and information engineering in Europe, Project Nr. 225997CP-1-2005-1-FR-ERASMUS-TNPP, http://www.eiesurveyor.org/

[12] http://www.clubeea.org/ 